Article

\title{
Variations in the Strength of Association between Food Neophobia and Food and Beverage Acceptability: A Data-Driven Exploratory Study of an Arousal Hypothesis
}

\author{
Sara R. Jaeger ${ }^{1}\left(\mathbb{D}\right.$, Sok L. Chheang ${ }^{1}$ and John Prescott ${ }^{2,3, *(D)}$ \\ 1 The New Zealand Institute for Plant \& Food Research Limited, Mt Albert Research Centre, \\ Auckland 1003, New Zealand; Sara.Jaeger@plantandfood.co.nz (S.R.J.); \\ sok.chheang@plantandfood.co.nz (S.L.C.) \\ 2 TasteMatters Research \& Consulting, Sydney, NSW 1230, Australia \\ 3 Department DAGRI, University of Florence, 50144 Florence, Italy \\ * Correspondence: prescott@taste-matters.org
}

\section{check for} updates

Citation: Jaeger, S.R.; Chheang, S.L.; Prescott, J. Variations in the Strength of Association between Food Neophobia and Food and Beverage Acceptability: A Data-Driven Exploratory Study of an Arousal Hypothesis. Nutrients 2021, 13, 3657. https://doi.org/10.3390/ nu13103657

Academic Editors: Paulina Nowicka and Pernilla Sandvik

Received: 19 September 2021

Accepted: 15 October 2021

Published: 19 October 2021

Publisher's Note: MDPI stays neutral with regard to jurisdictional claims in published maps and institutional affiliations.

Copyright: (c) 2021 by the authors. Licensee MDPI, Basel, Switzerland. This article is an open access article distributed under the terms and conditions of the Creative Commons Attribution (CC BY) license (https:/ / creativecommons.org/licenses/by/ $4.0 /)$.

\begin{abstract}
The negative impact of food neophobia (FN) on food and beverage (F\&B) liking extends beyond foods and beverages that are novel. In addition, F\&Bs that are high in flavour intensity, perceived as dangerous, or have connections to other cultures are likely to elicit rejection by those high in FN. Each of these factors have been established as producing increased arousal, potentially to an unpleasant degree. The aim of this study was to explore the hypothesis that increased arousal underlies all causes of rejection due to FN. To do this, we analysed and interpreted existing data based on online surveys that measured FN and liking for a broad range of F\&B names from 8906 adult consumers in the USA, United Kingdom, Australia, Germany and Denmark. Negative associations between FN and liking of varying strengths were evident for $90 \%$ of the F\&Bs. Consistent with the arousal hypothesis, F\&Bs (a) with high flavour intensity, whether produced by chilli, other spices, or flavours, (b) from other cultures, (c) often perceived as dangerous, or (d) that were novel or had novel ingredients showed the strongest negative relationships between FN and liking. Conversely, F\&Bs whose liking scores were only very weakly related to FN had low arousal characteristics: high familiarity, sweetness, mild flavours, strong connections to national food cultures, or some combination of these factors. Since this study was exploratory and conducted on existing data, there was no direct measure of arousal, but this is recommended for future, stronger tests of this arousal hypothesis.
\end{abstract}

Keywords: neophobia; arousal; liking; consumer research; product research; adults

\section{Introduction}

\subsection{Food Neophobia}

Typically defined as a relative reluctance to consume unfamiliar foods or beverages, food neophobia (FN) is recognised as an adaptive trait of omnivores, including humans. In children, a sensitive period beginning towards the end of the second year during which new foods are frequently rejected has been identified [1]. This period is generally considered to be a developmental stage that limits ingestion of unfamiliar, and therefore potentially dangerous, items that might be mistaken for food. FN in children has a significant impact on diet by reducing the number and variety of foods that are tried, as well as the range of foods that are preferred [2-4].

In adults, FN exists as a continuous trait on which the population is distributed. Based on scores on the Food Neophobia Scale (FNS) [5], the distribution ranges from 10 to 70-from neophilic to highly neophobic — with a positive skew, such that the bulk of the population is usually classified as having low to medium FN [6,7]. Nevertheless, some estimates from population samples in the USA based on a median split of FNS scores 
suggest that $40-45 \%$ of individuals are relatively high in FN [8]. As with children, high FN in adults is associated with reduced dietary variety and more disliked foods [9], reduced intention to try new foods [2], and negative attitudes towards foods originating from other cultures [10].

Although FN is defined, and prima facie measured, as a response to food novelty, there is evidence that high scores on the FNS also reflect rejection of, or lower preference for, foods that vary along dimensions other than familiarity. Thus, although food novelty is an issue for both children and adults high in FN [11], those with higher FN also give lower liking ratings to, and are less likely to consume or even try, many familiar foods $[2,6,9,12-17]$. They also show a reluctance to re-try foods that they have already tasted [15].

High FN has also been linked to differential responses to different types of foods, whether familiar or not. Thus, novel foods of animal origin (meat, seafood, eggs, dairy) tend to generate more negative responses than do novel vegetables, fruits or grains [18]. However, more recent research on large samples (>1000 people) divided according to FN has shown that all food types, novel or familiar, tend to be less acceptable in high FN individuals [6]. Moreover, this was true even for common, everyday consumables including meats, fruits and vegetables. In addition, increasing $\mathrm{FN}$ was associated with increasing numbers of disliked foods across all categories, although there was some evidence that seafood was most strongly disliked as FN increased.

The most frequent explanation of FN is based on the idea, at least in children, that avoidance of unknown foods reduces the risk of consuming potential toxins. It is therefore seen as an evolutionary adaptation in response to the Omnivore's Dilemma [19], an interpretation supported by the large genetic component to FN [20]. While this may be true in children, in the sense that novelty appears to be crucial, these findings suggest that initial responses to food novelty may become more generalised to a broader range of foods in adults, or that food novelty is not the only source of neophobic responses. What has not been determined is whether there is a common denominator, including, but not limited to, novelty of the foods and beverages that adults high in FN tend to reject or find less acceptable than those lower in FN.

\subsection{Food Neophobia and Arousal}

One potential defining feature of responses to F\&Bs in those who are high in FN is that they elicit unpleasant levels of arousal. In the psychological literature, arousal refers to a dimension that includes a complex of internal feeling states, autonomic activation and focussed attention [21,22]. When arousal is high, as in emotional states such as fear, the psychological and physiological reactions can be seen as responses to the perception of external threats. Berlyne [23] described the hedonic implications of arousal produced by sensory stimuli in terms of an inverted U-shape such that both low and high arousal were associated with low hedonic value, while hedonic maxima were reached at moderate levels of arousal. Key to this relationship were both the novelty and complexity of the stimuli. Thus, hedonic responses start off low-essentially boredom—for simple, familiar stimuli, rising to peak interest and enjoyment when complexity or novelty are moderate. Subsequent decreases in hedonic evaluations for highly complex and/or very unfamiliar stimuli suggest that these stimuli are the source of unpleasantly high arousal.

\subsection{Sources of Arousal in Foods}

Novelty per se is therefore a source of high arousal and there is evidence that this is due to the fact that unfamiliar foods are perceived as more potentially dangerous than are familiar foods [24]. However, high FN individuals actually appear to be hyperreactive to foods, irrespective of their novelty. This is seen in increases in physiological indices of arousal when viewing pictures of foods [25] or touching actual foods [26], and decreased sniffing of food odours [27] all independent of food familiarity. In addition to stimulus novelty, high arousal is also a function of complexity and intensity in sensory stimuli $[23,28,29]$. Thus, high FN is associated with reduced liking for, and choice of, 
foods that are intensely flavoured, e.g., those foods that are bitter, astringent or high in pungency $[12,13]$. This has been interpreted as due to higher arousal in response to inherent warning signals that foods may be dangerous to consume. Hence, foods that are strongly bitter induce stronger responses in physiological measures that reflect arousal [29]. The same effect on food acceptability appears to occur when foods are higher in complexity, such that bland foods were overall more acceptable to high FN individuals than were complex flavoured foods, while neophiliacs showed no such difference [30].

The potential role of high arousal in FN has been supported by experimental studies that have manipulated arousal and examined the impact on selection of novel foods. Pliner et al. [24] induced fear by informing participants that they would be giving an impromptu speech to other students, finding that this group chose fewer novel foods than a low fear group, at least when hunger was low. Manipulating arousal using a video game, Pliner and Melo [31] found that low arousal participants ate significantly more novel foods than when the game produced high arousal. Those who were high in the trait of seeking out new sensations-sensation seeking [32] — were much more likely to choose novel foods when arousal was low, but when arousal was high, the effect of sensation seeking on novel food choice was minimal.

\subsection{Food Neophobia and Sensory Sensitivity}

The relationship between FN and arousal may actually be a reflection of a more general heightened responsiveness to stimuli by those high in FN. This is evident in the close relationship between FN and more general (that is, not food-related) stimulus neophobia $[2,5,33]$. In children, high levels of negative emotional responses to novelty per se may be a risk factor for developing high FN [34]. In addition, high FN is associated with general trait anxiety [5] and lower sensation seeking [5,31,35]. FN also tends to cluster with other arousal-related personality traits including disgust sensitivity and sensitivity to punishment [36,37] when the impact of pungency on food choices is studied. There is even some evidence that FN is linked to aspects of social anxiety, in that high FN individuals seem less open to other cultures in general, not just in terms of their cuisines [38].

Indeed, both anxiety and a broad sensory sensitivity have been shown to be associated with selective/picky eating in children [39], and such sensitivity also appears to link anxiety and FN in young adults [40]. One consequence of such sensitivity may be attentional biases towards novelty in foods, which in children is evident, generally when presented with unfamiliar fruits and vegetables, but which is much stronger in those with high FN [41]. Such attentional biases towards threatening stimuli are a characteristic of anxiety disorders in adults [42].

\subsection{Research Aims and Approach}

Based on these earlier findings, the present research aimed to explore if relationships between FNS scores and F\&B acceptability ratings in an existing data set drawn from multiple countries was consistent with arousal as a unifying explanation for degree of neophobic response. We hypothesised that F\&Bs with pre-determined characteristics that potentially induced arousal would more likely be rejected or found less acceptable by adults higher in FN than by those lower in FN. Although we did not employ a direct measure of the arousal produced by these foods (or rather, their names), there is sufficient evidence, as noted above, that F\&B characteristics such as novelty (including foreignness), complexity, perceived dangerousness, and intensity are rejected by those high in FN and are associated more generally with increased arousal.

Specifically, we examined responses of consumers measured on the FNS, and from a variety of countries, to names of F\&Bs that were selected to vary along multiple dimensions including overall novelty, novel ingredients/unexpected combinations of ingredients in familiar foods, flavour intensity, perceived 'dangerousness' and being familiar but polarising. We expected that F\&Bs high on one or more of these dimensions would show reduced acceptability in those with high FN and hence be consistent with an explanation 
that implicated higher arousal. Hence, the study reported here consists of a hypothesisdriven exploration and interpretation of existing data. Eight consumer studies in five Western countries contributed to the research with responses from a total of 8906 adult participants (Table 1). The data were collected in online surveys over a two-year period and involved $219 \mathrm{~F} \& \mathrm{Bs}$. An important reason to use online surveys in FN research is to help overcome the low participation rates of those high in FN in central location tests [43].

Table 1. Overview of studies included in the research.

\begin{tabular}{|c|c|c|c|c|c|c|c|}
\hline Study & Country & Date & $\begin{array}{c}\text { FN Score } \\
\text { M (SD) }\end{array}$ & $\begin{array}{c}\text { F\&B } \\
\text { Stimuli } \\
\text { (\% Foods) }\end{array}$ & $\begin{array}{l}\text { Number of } \\
\text { Consumers }\end{array}$ & $\begin{array}{c}\text { Age Range } \\
\text { (Years) }\end{array}$ & $\%$ Male \\
\hline 1 & USA & April 2019 & 30.5 (12.1) & $26(100)$ & 1563 & $18-65$ & 50 \\
\hline 2 & USA & June 2020 & $33.8(12.4)$ & $18(100)$ & 594 & $18-65$ & 50 \\
\hline 3 & USA & June 2020 & $34.5(11.5)$ & $30(67)$ & 1522 & $18-65$ & 49 \\
\hline 4 & Australia & June 2018 & $31.5(10.8)$ & $42(81)$ & 758 & $18-69$ & 48 \\
\hline 5 & Australia & June 2020 & $33.3(11.5)$ & $18(100)$ & 1135 & $25-65$ & 49 \\
\hline 6 & UK & June 2020 & $31.4(11.7)$ & $20(95)$ & 1514 & $18-65$ & 47 \\
\hline 7 & Germany & June 2020 & $30.1(10.0)$ & $20(100)$ & 1040 & $18-65$ & 49 \\
\hline 8 & Denmark & Nov. 2019 & 32.7 (10.7) & $45(84)$ & 780 & $18-69$ & 51 \\
\hline
\end{tabular}

Notes: UK = United Kingdom; FN = food neophobia; $\mathrm{M}=$ mean and SD = standard deviation of summed FN score measured on the scale in Pliner and Hobden [5].

\section{Materials and Methods}

\subsection{Participants}

The participants were members of online panels managed by ISO accredited research providers. Table 1 shows the number of participants in each of the eight studies, their age range and male/female split. See Part 1 of Supplementary Materials for full participant details.

\subsection{Empirical Approach}

\subsubsection{Trait Food Neophobia}

The 10-item trait Pliner and Hobden Food Neophobia scale (FNS) [5] was used with 7 -point Likert scales $(1=$ 'disagree strongly' to $7=$ 'agree strongly'). In Study 1 , six of the ten items in Pliner and Hobden [5] were used, selected as those items that Ritchey, Frank, Hursti and Tuorila [44] identified as an appropriate subset for USA following a scale refinement study. In Study 2, 50\% of participants used the 10 -item FNS and $50 \%$ of participants used the 6-item FNS. In all studies, the presentation order of FN scale items was randomised.

\subsubsection{Product Names}

F\&B names were used as stimuli and enabled coverage of a large number of products. This was essential to achieve the research aim, and across the eight studies, a total of 219 names were used (full listing in Part 3 of Supplementary Materials).

While the selection of F\&B names in individual studies was partly subject to criteria determined by other research aims, care was taken to ensure diversity across the eight studies in accordance with several guiding principles. Foremost, stimuli with probable low /moderate hedonic appeal to neophobic individuals (e.g., dishes from other cultures, seafood/shellfish, offal, strong/spicy flavours) were included. There was a range of more/less familiar foods (e.g., 'instant noodles' vs. 'eggs Benedict' (USA); 'rice and milk porridge' vs. 'cheese fondue' (Denmark)). Foods and beverages were both represented, but the latter more infrequently, fitting with less diversity in this category. There was variation to capture breakfast, lunch and dinner F\&Bs (e.g., 'porridge/hot oatmeal', 'ham and cheese sandwich', 'prawn risotto'), as well as snack and sweet F\&Bs (e.g., 'mixed raw nuts', 'lemon mousse tart'), hot and cold F\&Bs (e.g., 'hot coffee', 'frozen yoghurt'), fruits and vegetables (e.g., 'apple', 'mixed green salad') and meat and vegetarian dishes (e.g., 'spaghetti Bolognaise', 'vegetable and bean hot pot'). Some stimuli contained novel ingredients 
(e.g., 'granola bar with insect flour') or combined known ingredients in untraditional ways (e.g., 'zucchini brownie', 'beef and beetroot sausages'). Some stimuli were only slightly different (e.g., 'apple and kale juice' vs. 'apple, orange and kale juice'; 'vegetarian pizza' vs. 'seafood pizza', 'tuna steak' vs. 'tuna pasta'). The F\&B names were developed by the authors and revised by sensory and consumer professionals with appropriate knowledge of eating and drinking habits in the different countries.

Consumers' liking responses were obtained on 9-point fully-labelled hedonic scale $(1=$ 'dislike extremely' to $9=$ 'like extremely') [45]. The F\&B names were presented according to a randomisation design.

\subsubsection{Data Collection and Previous Use}

Participants completed the survey in a location of their choosing. Stated liking data were always collected before FN. Demographic and socio-economic responses were obtained last. In all studies, data additional to those reported here were obtained, but not reported due to lack of relevance for the research aim.

The data for Studies 1 and 4 were previously used by Jaeger, Roigard, Hunter and Worch [46]. The data for Study 4 were also used by Jaeger, Roigard et al. [47], while those for Study 8 were used by Jaeger, Roigard, Ryan et al. [43]. However, these data have not previously been analysed collectively with a view to exploring arousal as a unifying explanation for neophobic responses.

\subsection{Data Analysis}

The same procedures were used in all studies, with analyses performed in XLSTAT v.2020.3.1 (Addinsoft, 2020) using a 5\% significance level. The data analysis strategy was borrowed from approaches used in several recent studies on related topics [43,46,48].

\subsubsection{Food Neophobia}

Following standard practice, summed scores (following reverse coding of required items) were calculated across all scale items, and in all studies, a continuous distribution of FN scores across the theoretically possible range (10 to 70) were observed. In Studies 1 and 2 , summed FN scores from the 6-item FNS were scaled to reflect the comparative values if 10 scale items had been used rather than 6 scale items (1.667 multiplier) (A comparison of summed scores from the two FNS sub-samples in Study 2 found these to have similar means based on the 6-item FNS ( $\mathrm{p}=0.10$ ) and supported a joint analysis). In all studies, values for Cronbach's alpha exceed the typical 0.7 threshold [49] to indicate acceptable internal consistency of the FN scale.

\subsubsection{Regression and Discretisation}

For each F\&B item, the relationship between FN and degree of liking/disliking was modelled using linear regression. Drawing directly on Jaeger et al. [43,46], the regressions were performed on mean values for liking calculated for each FN scale point ( $\mathrm{FN}=10$, $\mathrm{FN}=11, \mathrm{FN}=12$, etc.). These mean liking scores were based on different numbers of consumers due to the shape of the FN distribution (Part 4 of Supplementary Materials). Goodness of fit $\left(R^{2}\right)$, regression coefficients (b) (with 95\% confidence intervals) and intercepts (a) were retained for further evaluation. Of the 219 regression models, 199 (91\%) were significant at $p<0.05$ (Part 3 of Supplementary Materials lists those F\&B items where significant models were not established).

The 199 F\&Bs where a significant relationship was found between liking and FN were input to further analysis, seeking a meaningful reduction in this large number of individual F\&Bs that would enable hypothesis exploration. Accordingly, a discretisation procedure was applied to the distribution of the 199 regression coefficients to obtain an empirical separation of the F\&Bs into different groups based on the extent to which FN influenced average liking. The number of groups was determined by evaluating solutions from 4 to 12 and considering the relative frequency of F\&B names in each group and its interpretability. 
It was decided to retain six groups since $n \geq 7$ only resulted in ongoing sub-division of the two groups with least $F \& B$ items, and a more fine-grained distinction between $F \& B$ items where the regression coefficients were negative but very close to zero.

To facilitate the presentation of results, and, in turn, hypothesis exploration, names were assigned to the six groups of F\&Bs. These names were descriptive labels that differentiated the groups based on the direction and relative magnitude of the effect of FN on liking, as follows: "negative and very high" effect (18 F\&B names), "negative and high" effect (54 F\&B names), "negative and medium" effect (64 F\&B names), "negative and low effect" (34 F\&B names), "negative and very low effect" (27 F\&B names) and "positive and very low effect" (2 F\&B names) (Part 5 of Supplementary Materials provides a descriptive summary of the six groups).

\section{Results}

The present research was a hypothesis-driven exploration of arousal as a unifying explanation for degree of neophobic response, and we expected that F\&Bs with arousalinducing characteristics would be less acceptable to individuals higher in FN. The empirical results were interpreted against this background, focussing on groups of $F \& B$ items where F\&B liking decreased with FN.

\subsection{Characteristics of FEB Items in the Groups Where Strength of the Negative FN-Liking Relationship Differs}

By group, Table 2 lists the $197 \mathrm{~F} \& \mathrm{~B}$ names with negative regression coefficients in ascending order. They ranged from -0.132 ('Thai green chicken curry,' UK) to -0.002 ('Danish pastry,' DK), meaning that a 10-point increase in FN equates to a decrease in liking of, respectively, 1.3 and 0.02 scale points on the 9-point hedonic scale. This highlights the substantial effect of product category/type on the impact exerted by FN on liking (Part 6 of Supplementary Materials shows this visually).

To explore the arousal hypothesis as an explanation of the degree of neophobic response, the five groups of F\&Bs with negative regression coefficients are considered below, focussing on those characteristics that are shared among F\&B items in one or more group, but absent in other groups (Table 3). These categories of F\&B characteristics were derived by considering the items in the "very high" group and their unique characteristics. Progressing to the "high" group, new categories of F\&B characteristics were added to describe the F\&B items in this group not already captured by existing categories. The process continued in this manner until the "very low" group and any new categories needed to capture specific characteristics of its F\&Bs. The resulting categories of F\&B characteristics were diverse spanning aspects linked to sensory properties, ingredient combinations, product category, familiarity, cultural origins, etc. Multiple categories could apply to individual F\&B names.

The process of sequentially deriving the categories of $F \& B$ characteristics-progressing from the group where $F \& B$ items evoked the strongest neophobic responses to the group where F\&B items evoked the smallest neophobic response-was deliberate and aligned with the hypothesis of arousal as unifying explanation for F\&B dislike and rejection. Thus, we expected to uncover an ordering of the categories of $\mathrm{F} \& \mathrm{~B}$ characteristics from more to less arousal-inducing. 
Table 2. Listing of F\&B items ( $\mathrm{n}=197)$, sorted by group and regression coefficient (Coeff.) for effect of FN on liking/disliking. Names assigned to groups differentiate these on the level of negative effect of FN on F\&B liking. For visual clarity, grey shading is used to differentiate groups. The final column shows average liking ( $1=$ 'dislike extremely', $9=$ 'like extremely').

\begin{tabular}{|c|c|c|c|c|c|}
\hline Study & Country & F\&B Name & Coeff. & Group Name & Liking \\
\hline 6 & UK & Thai green chicken curry & -0.132 & Very High & 4.8 \\
\hline 4 & AU & Sushi & -0.128 & Very High & 6.3 \\
\hline 4 & AU & Thai green curry & -0.123 & Very High & 6.9 \\
\hline 6 & UK & Chilli chicken stir-fry & -0.122 & Very High & 5.2 \\
\hline 8 & DK & Sushi & -0.121 & Very High & 4.7 \\
\hline 5 & AU & Thai green curry & -0.120 & Very High & 5.4 \\
\hline 1 & USA & Sushi & -0.117 & Very High & 5.7 \\
\hline 7 & $\mathrm{DE}$ & Chilli chicken stir-fry & -0.116 & Very High & 5.7 \\
\hline 5 & AU & Sushi & -0.113 & Very High & 5.0 \\
\hline 6 & UK & Mild Indian curry (vegetarian) & -0.112 & Very High & 5.1 \\
\hline 6 & UK & Chilli con carne & -0.112 & Very High & 5.4 \\
\hline 6 & UK & Chicken korma & -0.109 & Very High & 5.4 \\
\hline 4 & AU & Prawn risotto & -0.109 & Very High & 6.0 \\
\hline 8 & DK & Thai meal & -0.106 & Very High & 6.1 \\
\hline 6 & UK & Spicy lamb meatballs & -0.106 & Very High & 4.9 \\
\hline 5 & $\mathrm{AU}$ & Prawn risotto & -0.104 & Very High & 5.2 \\
\hline 3 & USA & Spicy enchiladas & -0.103 & Very High & 5.5 \\
\hline 6 & UK & Lamb kebabs & -0.099 & Very High & 5.4 \\
\hline 1 & USA & Steamed mussels & -0.097 & High & 5.3 \\
\hline 7 & $\mathrm{DE}$ & Pasta with sundried tomato and garlic meat sauce & -0.095 & High & 5.8 \\
\hline 3 & USA & Vegetable chilli stir-fry & -0.095 & High & 5.0 \\
\hline 6 & UK & Chickpea salad (spicy) & -0.095 & High & 4.2 \\
\hline 7 & $\mathrm{DE}$ & Wholemeal pasta salad with chicken & -0.094 & High & 5.0 \\
\hline 7 & $\mathrm{DE}$ & Chicken and rice salad with spicy mayonnaise & -0.093 & High & 5.0 \\
\hline 3 & USA & Seafood pizza & -0.092 & High & 4.0 \\
\hline 5 & $\mathrm{AU}$ & Blue-vein cheese & -0.091 & High & 4.1 \\
\hline 7 & $\mathrm{DE}$ & Spinach and artichoke pizza & -0.091 & High & 4.4 \\
\hline 1 & USA & Fried oysters & -0.091 & High & 5.3 \\
\hline 3 & USA & Smoothie with avocado and almond milk & -0.090 & High & 4.3 \\
\hline 5 & AU & Spicy/hot chilli con carne & -0.090 & High & 4.9 \\
\hline 4 & AU & Bagel $\mathrm{w} /$ avocado and cream cheese & -0.090 & High & 6.3 \\
\hline 2 & USA & Shrimp taco & -0.087 & High & 6.0 \\
\hline 7 & $\mathrm{DE}$ & Salsa black bean burger & -0.087 & High & 3.5 \\
\hline 3 & USA & Chai latte & -0.087 & High & 4.4 \\
\hline 3 & USA & Kale, cucumber and apple juice & -0.087 & High & 4.3 \\
\hline 3 & USA & Stuffed crust pizza with cheese, tomato and shrimp & -0.086 & High & 4.8 \\
\hline 7 & $\mathrm{DE}$ & Vegetarian pizza & -0.086 & High & 4.6 \\
\hline 5 & $\mathrm{AU}$ & Tuna steak & -0.086 & High & 5.0 \\
\hline 1 & USA & Salsa poached eggs & -0.086 & High & 5.0 \\
\hline 3 & USA & Lasagna made with meat substitutes from pea protein & -0.085 & High & 4.1 \\
\hline 5 & $\mathrm{AU}$ & Strong mustard & -0.084 & High & 4.7 \\
\hline 3 & USA & Lentil and broccoli "meat balls" & -0.082 & High & 4.1 \\
\hline 3 & USA & Vegan "meat balls" made with soy protein & -0.082 & High & 4.0 \\
\hline 8 & DK & Kebab with green salad & -0.082 & High & 6.6 \\
\hline 5 & AU & Rabbit ragu & -0.082 & High & 3.7 \\
\hline 8 & DK & Toasted rye bread with fried egg and avocado & -0.081 & High & 6.3 \\
\hline 3 & USA & Granola bar with coconut and chia seeds & -0.080 & High & 5.1 \\
\hline 2 & USA & Fried mushrooms & -0.080 & High & 6.0 \\
\hline 3 & USA & Oat milk with cocoa flavour & -0.080 & High & 4.4 \\
\hline 2 & USA & Root vegetable stew & -0.080 & High & 5.1 \\
\hline 4 & $\mathrm{AU}$ & Spinach and tomato omelette & -0.079 & High & 6.7 \\
\hline 1 & USA & Refried beans & -0.078 & High & 6.3 \\
\hline 8 & DK & Vegetarian pizza & -0.078 & High & 5.1 \\
\hline 1 & USA & Three cheese and chorizo omelette & -0.078 & High & 6.4 \\
\hline 3 & USA & Kombucha with ginger & -0.077 & High & 3.7 \\
\hline 3 & USA & Onion and beet salad & -0.077 & High & 3.9 \\
\hline 3 & USA & Baked salmon & -0.077 & High & 5.8 \\
\hline 1 & USA & Hot pastrami sandwich & -0.077 & High & 6.6 \\
\hline 6 & UK & Chicken fried rice & -0.076 & High & 6.3 \\
\hline 3 & USA & Apple, orange and kale juice & -0.076 & High & 5.1 \\
\hline 5 & $\mathrm{AU}$ & Smoked cheese & -0.075 & High & 3.7 \\
\hline 1 & USA & Eggs Benedict & -0.075 & High & 6.3 \\
\hline 6 & UK & Tuna pasta & -0.074 & High & 5.2 \\
\hline 1 & USA & Kidney bean salad & -0.074 & High & 5.0 \\
\hline 3 & USA & Wholemeal pasta with garlic and tomato sauce & -0.074 & High & 5.9 \\
\hline 3 & USA & Breakfast burrito & -0.073 & High & 6.2 \\
\hline
\end{tabular}


Table 2. Cont.

\begin{tabular}{|c|c|c|c|c|c|}
\hline Study & Country & F\&B Name & Coeff. & Group Name & Liking \\
\hline 2 & USA & Seafood chowder & -0.073 & High & 5.7 \\
\hline 8 & DK & Roasted nuts & -0.072 & High & 6.6 \\
\hline 8 & DK & Pasta salad with feta cheese & -0.072 & High & 6.1 \\
\hline 4 & AU & Vegetable and bean hot pot & -0.072 & High & 6.5 \\
\hline 6 & UK & Soy milk & -0.071 & High & 3.5 \\
\hline 3 & USA & Vegetable pot pie & -0.071 & High & 5.4 \\
\hline 6 & UK & Sardines on toast & -0.070 & Medium & 3.8 \\
\hline 8 & DK & Mixed nuts with dried fruits & -0.070 & Medium & 6.0 \\
\hline 2 & USA & Corn chowder & -0.069 & Medium & 5.8 \\
\hline 8 & DK & Spinach and tomato omelette & -0.068 & Medium & 6.8 \\
\hline 6 & UK & Crackers with salmon pate & -0.068 & Medium & 4.4 \\
\hline 7 & $\mathrm{DE}$ & Stuffed bread with cheese and herbs & -0.068 & Medium & 6.1 \\
\hline 8 & DK & Vegetable juice & -0.067 & Medium & 4.9 \\
\hline 3 & USA & Zucchini brownie & -0.067 & Medium & 4.5 \\
\hline 1 & USA & Vegetable and bean casserole & -0.066 & Medium & 5.9 \\
\hline 6 & UK & Beef and beetroot sausages & -0.066 & Medium & 4.1 \\
\hline 4 & AU & Apple and kale juice & -0.066 & Medium & 5.3 \\
\hline 1 & USA & Baked salmon & -0.065 & Medium & 7.1 \\
\hline 6 & UK & Mixed green salad & -0.065 & Medium & 5.9 \\
\hline 7 & $\mathrm{DE}$ & Vegetarian meat loaf & -0.065 & Medium & 3.4 \\
\hline 6 & UK & Tuna steak & -0.065 & Medium & 5.5 \\
\hline 2 & USA & Lamb stew & -0.065 & Medium & 5.1 \\
\hline 4 & AU & Ham and tomato muffin & -0.064 & Medium & 6.3 \\
\hline 5 & AU & Pickled herring & -0.064 & Medium & 3.1 \\
\hline 3 & USA & Hot coffee & -0.063 & Medium & 6.3 \\
\hline 2 & USA & Lentil and beet soup & -0.063 & Medium & 4.6 \\
\hline 7 & $\mathrm{DE}$ & Ham and potato soup & -0.063 & Medium & 6.0 \\
\hline 8 & $\mathrm{DK}$ & Herbal tea & -0.063 & Medium & 4.5 \\
\hline 4 & AU & Caesar salad & -0.063 & Medium & 6.9 \\
\hline 4 & AU & Vegetarian sausages & -0.062 & Medium & 5.1 \\
\hline 1 & USA & Pickled beet and onion salad & -0.061 & Medium & 4.1 \\
\hline 8 & DK & Cheese fondue & -0.061 & Medium & 4.8 \\
\hline 2 & USA & Cream of mushroom soup & -0.061 & Medium & 5.7 \\
\hline 1 & USA & Breakfast burrito & -0.060 & Medium & 6.9 \\
\hline 8 & DK & Salmon and green salad & -0.058 & Medium & 6.6 \\
\hline 4 & $\mathrm{AU}$ & Frozen yoghurt & -0.058 & Medium & 6.2 \\
\hline 7 & $\mathrm{DE}$ & Chicken casserole & -0.058 & Medium & 6.2 \\
\hline 3 & USA & Club soda & -0.057 & Medium & 4.7 \\
\hline 4 & AU & Raw snack vegetables & -0.057 & Medium & 6.2 \\
\hline 7 & $\mathrm{DE}$ & Potato and lentil soup & -0.056 & Medium & 6.0 \\
\hline 7 & $\mathrm{DE}$ & Spicy red cabbage & -0.056 & Medium & 5.2 \\
\hline 4 & $\overline{A U}$ & I Yoghurt & -0.055 & Medium & 7.1 \\
\hline 4 & AU & Egg mayonnaise sandwich & -0.055 & Medium & 6.4 \\
\hline 2 & USA & Burger with patty from $100 \%$ plant-based meat & -0.055 & Medium & 5.0 \\
\hline 4 & AU & Mixed green salad & -0.055 & Medium & 7.0 \\
\hline 6 & UK & Dairy-free yoghurt & -0.054 & Medium & 4.4 \\
\hline 8 & DK & Beer & -0.054 & Medium & 5.3 \\
\hline 8 & $\mathrm{DK}$ & Sparkling water & -0.054 & Medium & 5.6 \\
\hline 1 & USA & Baked rabbit & -0.054 & Medium & 4.0 \\
\hline 1 & USA & Liver pate & -0.053 & Medium & 3.5 \\
\hline 5 & $\mathrm{AU}$ & Brussel sprouts & -0.053 & Medium & 5.0 \\
\hline 2 & USA & Veal burger & -0.053 & Medium & 4.9 \\
\hline 4 & $\mathrm{AU}$ & Hot coffee & -0.052 & Medium & 7.1 \\
\hline 1 & USA & Tripe and onions & -0.052 & Medium & 3.5 \\
\hline 3 & USA & Tossed green salad with red onions & -0.052 & Medium & 6.3 \\
\hline 8 & DK & Mixed grilled vegetables & -0.052 & Medium & 7.4 \\
\hline 2 & USA & Impossible ${ }^{\mathrm{TM}}$ burger (from plants) & -0.052 & Medium & 5.0 \\
\hline 3 & USA & Fish fingers & -0.052 & Medium & 5.3 \\
\hline 8 & $\mathrm{DK}$ & Stewed apples & -0.052 & Medium & 5.8 \\
\hline 4 & $\mathrm{AU}$ & Mixed raw nuts & -0.051 & Medium & 6.8 \\
\hline 7 & $\mathrm{DE}$ & Vegan bratwurst & -0.051 & Medium & 3.3 \\
\hline 4 & AU & Camomile tea & -0.051 & Medium & 5.2 \\
\hline 4 & AU & Iced coffee & -0.050 & Medium & 6.0 \\
\hline 3 & USA & Granola bar with insect flour & -0.050 & Medium & 3.1 \\
\hline 6 & UK & Savoury mince & -0.050 & Medium & 6.1 \\
\hline 8 & DK & Skyr with muesli & -0.050 & Medium & 5.1 \\
\hline 4 & AU & Croissant & -0.049 & Medium & 7.1 \\
\hline 8 & DK & Bun (Focaccia bread) with turkey, salad and dressing & -0.049 & Medium & 6.9 \\
\hline 1 & USA & Mixed green salad & -0.049 & Medium & 7.3 \\
\hline 8 & DK & Ham and cheese quiche & -0.048 & Medium & 6.9 \\
\hline
\end{tabular}


Table 2. Cont.

\begin{tabular}{|c|c|c|c|c|c|}
\hline Study & Country & F\&B Name & Coeff. & Group Name & Liking \\
\hline 2 & USA & Lamb chops & -0.047 & Low & 5.6 \\
\hline 7 & $\mathrm{DE}$ & Bread and cheese & -0.047 & Low & 7.1 \\
\hline 1 & USA & Stewed prunes & -0.045 & Low & 3.4 \\
\hline 7 & DE & Herring fillet in tomato sauce & -0.044 & Low & 5.2 \\
\hline 3 & USA & Strawberry flavoured milk (from cows) & -0.043 & Low & 5.4 \\
\hline 8 & DK & Quiche with leek and bacon & -0.043 & Low & 7.1 \\
\hline 8 & DK & Muesli with milk & -0.042 & Low & 5.4 \\
\hline 2 & USA & $\begin{array}{l}\text { Burger with patty from ground beef and vegetable } \\
\text { blend }(50: 50)\end{array}$ & -0.042 & Low & 5.4 \\
\hline 4 & AU & Cheese and vegemite sandwich & -0.042 & Low & 6.0 \\
\hline 4 & AU & Scrambled eggs & -0.041 & Low & 7.4 \\
\hline 2 & USA & Fried liver & -0.041 & Low & 3.9 \\
\hline 5 & $\mathrm{AU}$ & Dark chocolate & -0.040 & Low & 6.5 \\
\hline 8 & DK & White bread roll with ham and cheese & -0.040 & Low & 6.8 \\
\hline 1 & USA & Tuna salad sandwich & -0.039 & Low & 6.7 \\
\hline 8 & DK & Wholemeal bread with jam & -0.038 & Low & 6.4 \\
\hline 8 & DK & Fish cake on bread & -0.038 & Low & 7.0 \\
\hline 3 & USA & Strawberry and banana smoothie & -0.037 & Low & 6.8 \\
\hline 4 & $\mathrm{AU}$ & Sparkling water & -0.037 & Low & 5.7 \\
\hline 7 & $\mathrm{DE}$ & Spaghetti Bolognaise & -0.037 & Low & 7.4 \\
\hline 8 & $\mathrm{DK}$ & Rye bread with sliced meat & -0.036 & Low & 6.3 \\
\hline 1 & USA & Pickled pigs' feet & -0.036 & Low & 2.7 \\
\hline 6 & UK & Pork and potato sausages & -0.036 & Low & 5.4 \\
\hline 4 & $\mathrm{AU}$ & Peanut butter sandwich & -0.035 & Low & 6.4 \\
\hline 1 & USA & Chilli cheese dog & -0.035 & Low & 6.6 \\
\hline 6 & UK & Chicken casserole & -0.034 & Low & 6.7 \\
\hline 4 & AU & Porridge/hot oatmeal & -0.033 & Low & 6.2 \\
\hline 8 & DK & Rye bread with cheese & -0.033 & Low & 7.0 \\
\hline 4 & AU & Ham and cheese muffin & -0.033 & Low & 6.6 \\
\hline 2 & USA & All-American beef stew & -0.032 & Low & 7.0 \\
\hline 8 & DK & Beef rissole and potato salad & -0.031 & Low & 7.5 \\
\hline 4 & AU & Instant noodles & -0.031 & Low & 6.0 \\
\hline 4 & AU & Fresh fruit salad & -0.031 & Low & 7.4 \\
\hline 8 & DK & Water & -0.030 & Low & 7.8 \\
\hline 8 & DK & $\begin{array}{l}\text { Raw vegetables: tomato, cucumber, cauliflower, } \\
\text { capsicum }\end{array}$ & -0.030 & Low & 7.5 \\
\hline 4 & AU & \begin{tabular}{l|l} 
Lemon mousse tart \\
\end{tabular} & -0.028 & Very Low & 6.4 \\
\hline 4 & AU & Spaghetti Bolognaise & -0.027 & Very Low & 7.5 \\
\hline 3 & USA & Beef lasagna & -0.027 & Very Low & 7.3 \\
\hline 1 & USA & Cereal/muesli & -0.026 & Very Low & 6.4 \\
\hline 4 & $\mathrm{AU}$ & Banana & -0.026 & Very Low & 7.1 \\
\hline 8 & DK & Coffee & -0.026 & Very Low & 6.7 \\
\hline 5 & AU & Mild cheese & -0.026 & Very Low & 6.7 \\
\hline 8 & DK & Warm liver pate with mushrooms & -0.025 & Very Low & 7.4 \\
\hline 8 & DK & Porridge/hot oatmeal & -0.024 & Very Low & 5.6 \\
\hline 8 & DK & Fruit salad & -0.024 & Very Low & 7.4 \\
\hline 7 & $\mathrm{DE}$ & Meat loaf & -0.023 & Very Low & 6.8 \\
\hline 4 & AU & Cereal/muesli & -0.023 & Very Low & 6.9 \\
\hline 4 & AU & Cold sliced meats & -0.022 & Very Low & 6.8 \\
\hline 1 & USA & Blueberry muffins & -0.022 & Very Low & 7.5 \\
\hline 8 & DK & Broth with vegetables and meat balls & -0.022 & Very Low & 7.2 \\
\hline 5 & $\mathrm{AU}$ & White rice & -0.020 & Very Low & 6.7 \\
\hline 8 & DK & Soft boiled egg with bread & -0.020 & Very Low & 7.5 \\
\hline 5 & AU & Garlic bread & -0.020 & Very Low & 7.5 \\
\hline 4 & AU & Ham and cheese sandwich & -0.018 & Very Low & 7.1 \\
\hline 1 & USA & Instant noodles & -0.018 & Very Low & 6.2 \\
\hline 2 & USA & Chicken sandwich & -0.016 & Very Low & 7.7 \\
\hline 3 & USA & Spaghetti with tomato sauce & -0.015 & Very Low & 7.6 \\
\hline 4 & AU & Fruit juice & -0.014 & Very Low & 7.1 \\
\hline 1 & USA & Lasagna & -0.014 & Very Low & 8.0 \\
\hline 8 & DK & Spaghetti Bolognaise & -0.013 & Very Low & 8.0 \\
\hline 5 & AU & Apple & -0.011 & Very Low & 7.2 \\
\hline 8 & DK & Danish pastry & -0.002 & Very Low & 6.7 \\
\hline
\end{tabular}

Notes: The group of F\&B items with positive regression coefficients $(n=2)$ is not shown. The F\&B items with non-significant models $(\mathrm{n}=20)$ are not shown. AU $=$ Australia, $\mathrm{DE}=$ Germany, $\mathrm{DK}=$ Denmark 
Table 3. Derived categories of F\&B characteristics and their presence/absence in the five groups categorising the varying negative effect of FN of F\&B liking ("very high", "high", "medium", "low" and "very low"). The categories of F\&B characteristics should be seen in the context of those above it and refer to F\&B characteristics not previously captured. The exemplar F\&B items illustrate the associated category of F\&B item characteristics, but items may also fit into other categories without this being shown.

\begin{tabular}{|c|c|c|c|c|c|c|}
\hline \multirow{2}{*}{$\begin{array}{l}\text { Categories of F\&B } \\
\text { Characteristics }\end{array}$} & \multicolumn{5}{|c|}{ Degree of Negative Effect of FN on Liking } & \multirow[b]{2}{*}{ Exemplars } \\
\hline & $\begin{array}{l}\text { Very } \\
\text { High }\end{array}$ & High & Medium & Low & $\begin{array}{l}\text { Very } \\
\text { Low }\end{array}$ & \\
\hline From other cultures & $\mathrm{x}$ & $\mathrm{x}$ & & & & Thai green curry, chicken korma \\
\hline Shellfish/Sushi & $\mathrm{x}$ & $\mathrm{X}$ & & & & Prawn risotto, sushi \\
\hline Chilli/Spicy & $\mathrm{x}$ & $\mathrm{x}$ & $\mathrm{x}$ & & & Chilli con carne, spicy lamb meatballs \\
\hline Strong flavour & & $\mathrm{x}$ & $\mathrm{x}$ & & & $\begin{array}{c}\text { Blue cheese, kombucha with ginger, beet and } \\
\text { onion salad }\end{array}$ \\
\hline Unusual meat/Offal & & $\mathrm{x}$ & $\mathrm{x}$ & & & Rabbit, veal, liver, tripe and onions \\
\hline Fish & & $x$ & $x$ & $\mathrm{x}$ & & Sardines on toast, tuna steak, baked salmon \\
\hline Reduced familiarity & & $\mathrm{x}$ & $\mathrm{x}$ & $\mathrm{x}$ & & $\begin{array}{l}\text { Three cheese and chorizo omelette, } \\
\text { cheese fondue }\end{array}$ \\
\hline Familiar but often disliked & & $\mathrm{x}$ & $\mathrm{x}$ & $\mathrm{x}$ & & $\begin{array}{l}\text { Brussel sprouts, dark chocolate, coffee, } \\
\text { porridge, vegetable juice }\end{array}$ \\
\hline Familiar w / novel ingredients & & $\mathrm{x}$ & $\mathrm{x}$ & & & $\begin{array}{l}\text { Insect flour, chia seeds, oat milk, } 100 \% \\
\text { plant-based meat, soy protein }\end{array}$ \\
\hline $\begin{array}{l}\text { Familiar w/ unusual } \\
\text { ingredient/aspect }\end{array}$ & & $\mathrm{x}$ & $\mathrm{x}$ & & & $\begin{array}{l}\text { Apple and kale juice, zucchini brownie, } \\
\text { stewed apples }\end{array}$ \\
\hline Beans/Legumes & & $x$ & $x$ & & & $\begin{array}{l}\text { Vegetable and bean casserole, kidney } \\
\text { bean salad }\end{array}$ \\
\hline Vegetables/Salad & & & $\mathrm{x}$ & & & $\begin{array}{l}\text { Mixed green salad, mixed grilled vegetables, } \\
\text { raw snack vegetables }\end{array}$ \\
\hline Soup & & & $\mathrm{x}$ & & & $\begin{array}{l}\text { Corn chowder, ham and potato, cream } \\
\text { of mushroom }\end{array}$ \\
\hline Non-alcoholic beverages & & & $\mathrm{x}$ & $\mathrm{x}$ & $\mathrm{x}$ & $\begin{array}{l}\text { Water, fruit juice, club soda, strawberry and } \\
\text { banana smoothie }\end{array}$ \\
\hline Familiar hot meals w/ meat & & & $\mathrm{x}$ & $\mathrm{x}$ & $\mathrm{x}$ & Spaghetti Bolognaise, lasagna, meat loaf \\
\hline Familiar and grain-based & & & & $\mathrm{x}$ & $\mathrm{x}$ & $\begin{array}{l}\text { Ham and cheese sandwich, rye bread } \\
\text { with cheese }\end{array}$ \\
\hline Familiar desserts/cakes & & & & & $\mathrm{x}$ & Blueberry muffins, Danish pastry \\
\hline Fruit & & & & & $\mathrm{x}$ & Apple, banana \\
\hline Mild flavour & & & & & $\mathrm{x}$ & Mild cheese, white rice \\
\hline
\end{tabular}

\subsection{1. "Very High" Negative Effect of FN on F\&B Liking}

The $18 \mathrm{~F} \& \mathrm{~B}$ items in the group labelled as "very high" in reference to the negative effect of FN on liking shared one or more of the characteristics: From other cultures (curry, korma, Thai, kebab, enchilada), Chilli/Spicy ('chilli con carne,' 'chilli chicken stir-fry,' 'spicy lamb meatballs'), and Shellfish/Sushi ('sushi,' 'prawn risotto'). F\&B items with explicit mention of chilli and spicy were found in both this cluster (i.e., "very high") and the "high" group, but items that explicitly or indirectly referenced "curry" were only found in this group ('Thai green chicken curry,' 'chicken korma'), and notably, this extended to 'mild Indian curry (vegetarian)' (UK). 'Sushi' featured in four studies and three countries (US, $\mathrm{AU}, \mathrm{DK})$, and the $95 \%$ confidence intervals around the regression coefficients overlapped, suggesting that the same highly negative effect of FN was country independent. There were no beverages in the "very high" group.

\subsection{2. "High" Negative Effect of FN on F\&B Liking}

The categories of F\&B characteristics that defined membership of the "very high" group-From other cultures, Chilli/Spicy and Shellfish/Sushi-were also represented in the "high" cluster (e.g., 'breakfast burrito' (US), 'chicken and rice salad with spicy mayonnaise' (DE) 'steamed mussels' (US)), although there were only three F\&B items in this cluster that explicitly mentioned chilli or spicy/hot, and these were all placed in the top quartile of the group according to the value of the regression coefficient.

The number of F\&B items in the "high" group - 54-was much larger than the "very high" group and considerably more diverse, leading to additional categories of F\&B characteristics (Table 3). The first of these-Strong flavour — referred to strong flavours other 
than chilli/spicy in accordance with the "hierarchical" coding process described above, and F\&B items representing this category were 'strong mustard' (AU), 'blue-vein cheese' $(\mathrm{AU})$, 'smoked cheese' (AU) and 'pasta with sundried tomato and garlic meat sauce' (DE).

The category named Dish with reduced familiarity were introduced in this group to encompass F\&B items with reduced familiarity that were not already covered by existing categories including From other cultures. Exemplar F\&B items were 'cheese fondue' (DK) and 'three cheese and chorizo omelette' (US). Additional new categories relating to higher/lower familiarity were: Familiar FEB from novel ingredients ('oat milk with cocoa flavour' (US), 'vegan meat balls made with soy protein' (US), 'granola bar with coconut and chia seeds' (US)), Familiar FEB but often disliked (e.g., 'fried mushrooms' (US), 'wholemeal pasta salad with chicken' (DE), 'root vegetable stew' (US)), and Familiar FEB with unusual ingredients/aspect (e.g., 'apple, orange and kale juice' (US), 'toasted rye bread with fried eggs and avocado' (DK) and 'roasted nuts' (DK)).

The "high" group included nine items containing shellfish or fish, and six of these had regression coefficients that placed them in the top half of the group (i.e., closer to the "very high" than to the "medium" group): 'steamed mussels' (US), 'seafood pizza' (US), 'fried oysters' (US), 'shrimp taco' (US), 'stuffed crust pizza with cheese, tomato and shrimp' (US) and 'tuna steak' (AU, UK)). In comparison, the remaining three items ('baked salmon' (US), 'tuna pasta' (UK) and 'seafood chowder' (US)) were more familiar and/or part of dishes where the fish/seafood flavour was a less dominant component. Beans/Legumes (e.g., 'refried beans' (US), 'kidney bean salad' (US), 'salsa black bean burger' (DE)) and Unusual meat/Offal (e.g., 'rabbit ragu' (AU)) were the final two categories of F\&B characteristics created to describe the items in this group.

\subsection{3. "Medium" Negative Effect of FN on F\&B Liking}

The largest group of F\&B items $(n=64)$ was extremely diverse (Table 3$)$ and drew further attention to the fact that the negative effect of FN on liking is pervasive rather than being limited to strictly novel foods and beverages. F\&B items which explicitly mentioned chilli were absent, and only one item mentioned spicy ('spicy red cabbage' (DE)). Connotations to other cultures were most obvious in 'breakfast burrito' (US), but also implied in 'pickled herring' (AU) and 'skyr with muesli' (DK) (skyr is an Icelandic variant of yoghurt). Items with Strong Flavour (other than chilli/spicy) such as 'pickled beet and onion salad' (US) and 'tossed green salad with red onions' (US), were infrequent also. These findings combined with the absence of Shellfish/Sushi items in the "medium" group highlighted its difference relative to the "very high" and "high" groups, and, more generally, how the negative effect of FN, although weaker, was still systematically linked to various categories of $\mathrm{F} \& \mathrm{~B}$ characteristics.

Distinct from the category Shellfish/Sushi, there were seven F\&B items with Fish in the "medium" group-'sardines on toast' (UK), 'crackers with salmon pate' (UK), 'baked salmon' (US), 'tuna steak' (UK), 'pickled herring' (AU), 'salmon and green salad' (DK) and 'fish fingers' (US). Among the other product specific categories, Unusual meat/Offal was represented by: 'liver pate' (US), 'tripe and onion' (US), 'baked rabbit' (US) and 'veal burger' (US)), and the former two items, such as 'pickled herring' probably placed in this lower-than-expected group due to widespread disliking (Table 3). New product-focussed categories of F\&B characteristics identified in the "medium" group were Vegetables/Salads, represented by 'mixed green salad' (US, UK, AU), 'Caesar salad' (AU), 'mixed grilled vegetables' (DK), 'raw snack vegetables' (AU)) and Soup. The latter appeared as a category that was largely specific to the "medium" group: 'lentil and beet soup' (US), 'cream of mushroom soup' (US), 'corn chowder' (US), 'potato and lentil soup' (DE) and 'ham and potato soup' (DE). However, there was a partial overlap with Beans/Legumes, which likely exerted a notable negative effect, based on the results for the "high" group.

The categories of F\&Bs' characteristics relating to familiarity/novelty were similar to those identified in the "high" group. F\&B items representing the category Dish with reduced familiarity were: 'stuffed bread with cheese and herbs' (DE), 'cheese fondue' (DK), 
'lamb stew' (US) and 'savoury mince' (UK). Extending from there: Familiar FEB from novel ingredients (e.g., 'burger with patty from $100 \%$ plant-based meat substitute' (AU), 'dairyfree yoghurt' (UK), 'granola bar with insect flour' (US)), Familiar FEB but often disliked (e.g., 'vegetable juice' (DK), 'herbal tea' (DK), 'stewed apples' (DK), 'beer' (DK), 'hot coffee' (US), 'Brussel sprouts' (AU), 'mixed nuts with dried fruits' (DK)) and Familiar FEB with unusual ingredients/aspects (e.g., 'zucchini brownie' (US), 'beef and beetroot sausages' (UK), 'apple and kale juice' (AU), 'ham and tomato muffin' (AU), 'vegetarian meat loaf' (DE), 'iced coffee' (AU), spinach and tomato omelette' (DK) and 'stuffed bread with cheese and herbs' (DE)). Familiarity for other items was reduced due to inclusion of a less known ingredient (e.g., 'bun (Focaccia bread) with turkey, salad and dressing' (DK) and 'skyr (Icelandic yoghurt) with muesli' (DK)).

A number of F\&B items-'sparkling water' (DK), 'club soda' (US), 'yoghurt' (AU), 'chicken casserole' (DE) and 'egg mayonnaise sandwich' (AU)—did not appear to fit within the categories of F\&B characteristics that defined membership of the "medium" group. However, new categories formed in the interpretative process for the "low" cluster did appear to represent several of these items, and it was unclear why the negative effect of FN was not lower.

\subsection{4. "Low" Negative Effect of FN on F\&B Liking}

In this group $(n=34)$, the absence of several of the categories of F\&B characteristics observed in the three previous groups was noted (Table 3). There were no F\&B items which directly identified them as being from other cultures, and there was only a single instance of reference to chilly/spicy: 'chilli cheese $\mathrm{dog}^{\prime}$ which is a relatively common food in the US. Neither were there any F\&B items representing the categories Strong Flavour, Shellfish/Sushi and Unusual meat/Offal. There were three Fish items, although 'herring fillet in tomato sauce' (DE) and 'fish cake on bread' (DK) are highly familiar and widely consumed, which is also true of 'tuna salad sandwich' (US). In the latter, the fish taste is also likely masked by other ingredients such as mayonnaise, making it more widely acceptable. The category capturing Familiar FEB with novel ingredients was missing from the "low" group, as were items containing Beans/Legumes. There was only a single F\&B item representing the Vegetable/Salad category-'raw vegetables: tomato, cucumber, cauliflower, capsicum' (DK).

Items representing the categories of $\mathrm{F} \& \mathrm{~B}$ characteristics named Familiar FEB but often disliked and Familiar FEB with unusual ingredient/aspect were well represented in the "low" group. The former included: 'cheese and vegemite sandwich' (AU), 'dark chocolate' (AU), 'wholemeal bread with jam' (DK), 'peanut butter sandwich' (AU), 'lamb chops' (US), 'quiche with leek and bacon' (DK) and 'porridge/hot oatmeal' (AU). Representatives of the latter category were: 'white bread roll with ham and cheese' (DK), 'burger with patty from ground beef and vegetable blend (50:50)' (US), 'strawberry flavoured milk (from cows)' (US) and 'pork and potato sausages' (UK).

Additional categories of F\&B characteristics emerged clearly in the "low" group: $F a$ miliar hot meals with meat and Familiar and grain-based (cold). The former included: 'spaghetti Bolognaise' (DE), 'chicken casserole' (UK), 'All-American beef stew' (US) and 'beef rissole and potato salad' (DK). The latter included: 'bread and cheese' (DE), 'muesli with milk' (DK), 'ham and cheese muffin' (AU), 'rye bread with sliced meat' (DK) and 'rye bread with cheese' (DK). Several popular non-alcoholic cold beverages were also found in this cluster: 'sparkling water' (AU), 'water' (AU) and 'strawberry and banana smoothie' (US).

The unexpected placement of 'fried liver' (US) and 'pickled pigs' feet' (US) in the "low" group can be attributed to widespread dislike, irrespective of degree of FN. For example, the estimated average liking for 'pickled pigs' feet' was lower than 3 on the 9-point scale even among the most neophilic participants. A similar explanation applies to 'stewed prunes' (US). 


\subsection{5. "Very Low" Negative Effect of FN on F\&B Liking}

Fitting the earlier patterns, the $27 \mathrm{~F} \& \mathrm{~B}$ items in the group where FN exerted a "very low" negative effect on F\&B liking were very diverse. The findings from the "low" group in relation to the absence of certain categories of F\&B characteristics largely replicated: from other cultures, chilli/spicy, strong flavour, shellfish/sushi, fish, unusual meat/offal, novel ingredients, beans/legumes, vegetables/salad and familiar with unusual ingredients/aspects. The only exceptions were 'garlic bread' (AU) and 'warm liver pate with mushrooms' (DK). For 'garlic bread', it seems plausible that Australian participants based on commercial offerings did not consider this to have Strong flavour. In Denmark, warm liver pate with mushroom is a highly popular topping on open sandwiches (smørrebrød) and common on weekend lunches.

Items fitting into categories of F\&B characteristics also observed in the "low" group were: Familiar hot meals with meat (e.g., spaghetti Bolognaise' (AU, DK), 'beef lasagna' (US), 'meat loaf' (DE) and 'chicken sandwich' (US; aka, chicken burger)), Familiar and grain-based (cold) (e.g., 'cereal/muesli' (US)), 'ham and cheese sandwich' (AU) and Familiar FEB but often disliked (e.g., 'coffee' (DK) and 'soft boiled egg with bread' (DK)).

Categories of F\&B characteristics not apparent in the previous groups were: Familiar desserts/cakes ('lemon mousse tart' (AU), 'blueberry muffin' (AU) and 'Danish pastry' (DK)), Fruit ('apple' (AU), 'banana' (AU)) and Mild flavour ('mild cheese' (AU), 'white rice' (AU) and 'cold sliced meats' (AU)).

\section{Discussion}

Food neophobia, with its negative consequences for food enjoyment and dietary quality $[6,9,50]$, has attracted much scholarly interest. Yet, even though it has become increasingly apparent that novelty is neither necessary nor sufficient to explain the food rejections of those high in $\mathrm{FN}$, an alternative common denominator of the F\&B characteristics that adults high in FN find less acceptable has not been established. In the current data set, liking for the vast majority of the $219 \mathrm{~F} \& \mathrm{~B}$ items, across a variety of countries, was negatively associated with FN, thus supporting conclusions from previous studies that FN in adults is not related only to novelty but also encompasses foods that are familiar [6]. Lower preferences for, and consumption frequency of, common food items therefore require a consideration of what factors other than novelty might also be involved. In the present research, we explored if there was evidence that arousal—specifically, unpleasantly high arousal-could be a likely candidate.

This hypothesis was developed taking into account evidence that foods per se are generally more arousing for those high in FN. This is evident in measures of arousal when viewing pictures of foods [25] or touching foods [26], as well as a general wariness when sniffing food odours [27]. It is possible, therefore, that foods and eating are more frequently associated with anxiety for those high in FN, perhaps due to fear that they may encounter an unfamiliar or unpleasant taste [24]. Consistent with this notion, experiencing foods tends to reduce the impact of FN on preferences whether the food is initially unfamiliar or not $[17,24]$.

\subsection{Arousal and Neophobic Responses}

Although the original purpose of collecting these data was not to test a hypothesis about the effects of F\&B characteristics on arousal, the results are consistent with our post hoc hypothesis that arousal may be a substrate for F\&B disliking and rejection. If our hypothesis had no explanatory value for neophobia-based food rejections, then we would expect that liking for familiar F\&Bs with intense flavours or links to other cultures, for example, would show no relationship with FN. Clearly, this was not the case.

The F\&B items in the group where the negative regression coefficients between liking and FN were "very high" — and to a lesser extent the F\&B items in the "high" groupimplicated all of the expected arousal-inducing categories of F\&B characteristics: flavour intensity whether produced by chilli, other spices or flavours, foods from other cultures 
(even if familiar), and the novelty of a dish or its ingredients. This is consistent with some general categories of arousal elicitation-particularly, intensity and novelty-that have been previously described $[12,13,23,28,29]$. Many items within these overlapping categories of F\&B characteristics may also elicit arousal due to their perceived complexity, although we have no way of estimating this from the present data. Some seafood items were also present in these groups of high negative effect of FN on liking. This should be viewed in the light of evidence that seafood is commonly considered to contain inherent risks (e.g., contaminants) in its consumption [51-54], and is therefore considered dangerous, and thus arousal-inducing, relative to other common F\&Bs.

Of relevance to the idea of arousal as a unifying factor was the fact that these findings (Table 2), especially for the "very high" group, were relatively uniform across different countries. It has previously been noted that preferences for relatively bland foods-bread, rice, potatoes - tend to be unaffected by FN [6]. This was replicated here, a finding that is also consistent with our arousal hypothesis. Those F\&Bs whose liking scores were only very weakly related to FN possessed characteristics not expected to induce arousal: high familiarity, sweetness, mild flavours, strong connections to national food cultures, or some combination of these factors. Additionally, it has been suggested [55] that food with high energy content-often sweet or high in fat, such as more common in the "very low" group - may be less likely to be perceived as unsafe and hence be limited in their ability to elicit neophobic responses because of their potential survival value.

\subsection{FEB Characteristics and Neophobic Responses}

While there was evidence to support arousal as a unifying explanation for F\&B rejection and dislike by high FN individuals, this explanation does not necessarily account for all the observed results. In between the extremes of the "very high" and "very low" groups, seemingly without an obvious linkage to arousal, a broad range of categories of F\&B characteristics were associated with some degree of neophobic response (Table 2). This could suggest that F\&B characteristics other than those addressed by the extant literature, chiefly novelty, complexity and intensity $[2,13,30]$, are at play, and/or that increased arousal explains strong neophobic responses, but not neophobic responses of intermediate strength. The F\&B items in those groups where the negative effect of FN on liking was only moderate ("medium" and "low") were also less uniform across cultures, as might be expected if culture-specific reasons for rejection were more influential in these groups. Alternatively, the low arousal associated with simple, familiar and low intensity foods might be a source of boredom in those low-moderate in FN [56] but could represent sought-after characteristics for the high FN individual.

Although the categories of $F \& B$ characteristics rather than the individual items herein were the key to addressing the research aim, it is appropriate to comment on the strength of the FN-liking relationship for F\&Bs when notably different to expectations. These "discrepancies" might reflect factors other than FN exerting an influence on liking. As one example, the regression coefficient for 'prawn risotto' (AU) meant that this item fitted in the "very high" group despite its ingredients not being unfamiliar, especially exotic or strongly flavoured, although there may be unfamiliarity in the sense that it may not be commonly eaten (see also earlier comments regarding seafood). In addition, in the UK, curries are highly familiar dishes that are no longer especially associated with other cultures, and yet 'mild vegetarian curry (vegetarian)' and 'chicken korma' were both in the "very high" group for this UK sample. However, some people still find curry of any sort too spicy, and this is probably a function of several things including FN, but also sensory sensitivity, as demonstrated by the reported close relationship of sensitivity to perception of pungency and rejection of pungent foods [37].

These and other discrepancies could also point to differences between the way familiarity and novelty in F\&Bs are operationally defined here, and the way in which these qualities are perceived by consumers. Thus, certain stereotypical associations may be influential with consumers. For example, based on its regression coefficient, 'chicken fried 
rice' (UK) was placed into the "high" group despite not appearing to meet the derived criteria for membership (i.e., no seafood and not intensely flavoured nor novel in its ingredients). However, fried rice is a popular component of many East Asian and Southeast Asian cuisines with origins in China [57], pointing to a likely perception of 'chicken fried rice' as exotic, and hence potentially challenging.

Based on regression coefficients for the relationship between FN and liking, some items were placed in groups of lower strength than was expected. We propose that such weaker relationships could reflect poor acceptability generally, potentially obscuring any effect of degree of FN. For example, 'pickled herring' (AU) might have been expected to fit in one of the two "high" groups considering its strong flavour. However, a likely explanation for why it placed in the "medium" group was the low average liking for 'pickled herring' (Section 3.1; Table 2). If an item is generally widely disliked, then the potential for FN to exert a large negative effect (i.e., have a large negative regression coefficient) is reduced. For 'sardines on toast' (UK) which also placed in the "medium" strength group, a different explanation seemed likely. Considering its strong flavour, placement in the "high" strength group could have been expected, but the long history of eating sardines in the UK (www.foodsofengland.co.uk, accessed on 20 June 2021) may have exerted an influence in terms of high familiarity.

Considering the inductive process whereby the categories of $F \& B$ characteristics (Table 3) were derived and the dependence of these categories on the items included in the research, it is necessary to acknowledge that they may lack interpretative value in relation to the relationship between FN and liking. The category Soup conveniently captured a property that several items had in common, but it is not clear how soup connects to neophobic response since the category spanned from 'seafood chowder' (US) which was included in the "high" group and 'broth with vegetables and meatballs' (DK) which was included in the "very low" group. Another caution regarding the categories of F\&B characteristics is that they are not complete in the sense of providing full coverage of major product classes, e.g., [58]; fruit is missing, for example. This was a consequence of low representation among the $219 \mathrm{~F} \& \mathrm{~B}$ items, even though fruit preference and consumption is influenced by degree of neophobia [6]. In particular, it is possible to imagine regression coefficients corresponding to a "medium" strength relationship between liking and FN for fruit that have intense flavours and/or are from other cultures (e.g., durian, gooseberries, pomegranate, mangosteen).

An important consideration in interpreting these data is whether factors other than increases in arousal could account for the observed relationships between FN and liking. The most obvious alternative explanation is that the relationship with FN is with the names of the F\&Bs, which may be unfamiliar to greater or lesser degrees. Certainly, many items in the "very high" group are, in some sense, more "exotic", especially in contrast to those F\&Bs in the "low" groups, which might be considered more mundane. So, are the "very high" F\&B items simply more novel/unfamiliar names? This seems unlikely. For example, given the influx of Asian and Indian restaurants throughout the UK and other Western countries in recent decades, dishes such as chilli chicken stir-fry, mild Indian curry or lamb kebabs are all well known. The same is certainly true of sushi in both the US and Australia. It is relevant to this issue that the data collection was conducted mostly on urban consumers in Western countries, where ethnic restaurants have been common for many years.

\subsection{Limitations and Suggestions for Future Research}

A post hoc interpretation of data that has been collected prior to hypothesis formulation is always likely to raise as many questions as it answers. We regard this as a positive in that it can lead to more explicit hypotheses in later studies. Nevertheless, the results of this exploratory and data-driven analysis should be interpreted with several caveats. The most obvious hurdle faced in this analysis is the absence of a formal measure of arousal. As noted earlier, we believe that our interpretation of responses is consistent with high arousal 
as an explanation for decreased liking for many foods with increasing FN. However, as a strict test of the arousal hypothesis of FN, future studies ought to include such a measure. Data collection via internet survey, as in the present data set, obviously precludes many of the laboratory-based measures of arousal, e.g., physiological monitoring. Possibly the most practical way of assessing arousal would be to collect ratings on emotions, in addition to those of liking, for each food. It is well established that the underlying structure of emotional terms involves valence and arousal as independent dimensions [59]. Measures of specific emotions such as fear, anxiety, alertness, all of which have a high arousal component, may therefore be highly suitable as direct measures [60]. Another possibility would be to use a measure such as the Affect Grid [22] which contains measures of both arousal and valence, in place of ratings of liking.

The F\&B items themselves also do not always provide a straightforward interpretation of the source of high arousal, and it is possible that a more systematic selection of $F \& B$ items may have helped in attributing the strength of the relationship between FN and liking to specific F\&B characteristics. In the group where FN had a "very high" negative impact on liking, it is possible that a given F\&B item was rejected due to it being from another culture, or being spicy, or otherwise having a strong flavour, or a combination of all three factors. In future research, a more systematic variation in F\&B characteristics based on arousal potential could help to disentangle this interdependency by creating $F \& B$ stimuli that a priori are expected to be associated with strong or weak neophobic responses. A structured approach to stimulus development would also allow for testing of the idea that a food that combines several characteristics typically associated with an "intermediate" neophobic response-perhaps a familiar food with multiple unusual ingredients-may fall into the "very high" group.

Our interpretations relied to a large extent on the discretisation process, which created group boundaries along a continuum from "very high" to "very low" negative impact of FN on F\&B liking. While arbitrary, the resulting groups of F\&B items nonetheless served as an operational means of describing features that allowed us to identify possible reasons for the relative degrees of rejection of individual F\&B items. Other approaches to creating groups of F\&B items could have been used, including expert judgment and/or input from consumers in each country. Our placement of individual F\&B items into Table 3 is also open to discussion and revision. The inductive process where categories of F\&B characteristics were developed and associated with different items was partly driven by the F\&B items included in the research and if other stimuli had been used, different categories may have arisen. For example, it is possible to imagine categories for national cuisines and had there been a category for Italian Food, 'prawn risotto' may have fitted there instead of being placed in the Shellfish/Sushi category. For completeness, we note that the absolute values of the regression coefficients between FN and liking for individual F\&B names should only be directly compared to other studies with caution since the use of written stimuli, online surveys, and analysis based on aggregate liking values for each FNS scale point all may influence the absolute values.

Finally, the research was conducted in five Western countries, but not designed to specifically address cross-cultural differences in FN, and the selection of F\&B items was not made with a view to country-to-country comparison. Rather, in keeping with an exploratory research strategy, a large number of different F\&B items which varied in their arousal-inducing potential were included, even if these items occurred in a single country only. Future research could address the important issue of cross-cultural differences more systematically using, for example, items that are consumed (or avoided) in many different countries, such as coffee, sushi, beef burger, pizza, cereal/muesli, apples, bread, eggs, potatoes, chocolate cake, water, beer, Brussel sprouts, liver, etc. A particular point of interest would be to establish how the uniformity of strong neophobic responses in the "very high" cluster evolves in different countries, and how the F\&B items become more diverse as the association between FN and food liking weakens. 


\section{Conclusions}

The present large data set provides a highly detailed, cross-cultural view of neophobic response to a variety of F\&Bs that varied in multiple ways. We hypothesised that food characteristics that potentially induced arousal would be more likely to be rejected as FN increased, and that arousal may act as a unifying explanation for degree of neophobic response. The empirical evidence, consistent with this notion, identified strong negative effects for F\&Bs with high flavour intensity whether produced by chilli, other spices or flavours, foods from other cultures, and the novelty of a dish or its ingredients. Additionally consistent with the arousal hypothesis was the finding that F\&Bs whose liking scores were only very weakly related to FN possessed characteristics not expected to induce arousal: high familiarity, sweetness, mild flavours, strong connections to national food cultures, or some combination of these factors. To overcome the limitations of the exploratory and data-driven approach, a range of suggestions for future research were proposed, notably direct measures of arousal, a more systematic selection of $F \& B$ items to be able to attribute the strength of the relationship between FN and liking to specific F\&B characteristics. It would also be very pertinent to address the important issue of cross-cultural differences more systematically using, for example, items that are consumed (or avoided) in many different countries.

Supplementary Materials: The following are available online at https:/ /www.mdpi.com/article/ 10.3390/nu13103657/s1. Table S1a-h: Summary of participant characteristics by study; Table S2: F\&B names used in Germany (Study 7) and Denmark (Study 8) in original languages; Table S3: Regression statistics for F\&B names included in the research and average hedonic scores $(n=219)$; Figure S1: Distribution of FN scores in Study 1; Table S4: Overview of the six groups of F\&B names with variable impact of FN on liking; Figure S2: Plot of average F\&B liking as a function of FN for selected F\&B names.

Author Contributions: S.R.J.: Conceptualization, Methodology, Formal Analysis, Writing-Original draft, Writing-Review and Editing. S.L.C.: Investigation, Data Curation. J.P.: Conceptualization, Writing-Original draft, Writing-Review and Editing. All authors have read and agreed to the published version of the manuscript.

Funding: This research was funded from The New Zealand Ministry for Business, Innovation and Employment and The New Zealand Institute for Plant and Food Research Limited.

Institutional Review Board Statement: The study was conducted according to the guidelines of the Declaration of Helsinki. All studies were covered by a general approval for sensory and consumer research from the Human Ethics Committee at The New Zealand Institute for Plant and Food Research Limited. Participants were assured that their responses would remain confidential. Reward points and promotional offers were offered as compensation.

Informed Consent Statement: Informed consent was obtained from all subjects involved in the study.

Data Availability Statement: The data presented in this study are available on request from the corresponding author.

Acknowledgments: Staff at the Sensory and Consumer Science Team at PFR are thanked for help in data collection.

Conflicts of Interest: The authors declare no conflict of interest. The funders had no role in the design of the study; in the collection, analyses, or interpretation of data; in the writing of the manuscript, or in the decision to publish the results.

\section{References}

1. Cashdan, E. Adaptiveness of food learning and food aversions in children. Soc. Sci. Inf. 1998, 37, 613-632. [CrossRef]

2. Hursti, U.-K.K.; Sjoden, P.-O. Food and General Neophobia and their Relationship with Self-Reported Food Choice: Familial Resemblance in Swedish Families with Children of Ages 7-17 Years. Appetite 1997, 29, 89-103. [CrossRef]

3. Russell, C.G.; Worsley, A. A Population-based Study of Preschoolers' Food Neophobia and Its Associations with Food Preferences. J. Nutr. Educ. Behav. 2008, 40, 11-19. [CrossRef] [PubMed] 
4. Skinner, J.D.; Carruth, B.R.; Wendy, B.; Ziegler, P.J. Children's food preferences: A longitudinal analysis. J. Am. Diet. Assoc. 2002, 102, 1638-1647. [CrossRef]

5. Pliner, P.; Hobden, K. Development of a scale to measure the trait of food neophobia in humans. Appetite 1992, 19, 105-120. [CrossRef]

6. Jaeger, S.R.; Rasmussen, M.A.; Prescott, J. Relationships between food neophobia and food intake and preferences: Findings from a sample of New Zealand adults. Appetite 2017, 116, 410-422. [CrossRef] [PubMed]

7. Monteleone, E.; Spinelli, S.; Dinnella, C.; Endrizzi, I.; Laureati, M.; Pagliarini, E.; Sinesio, F.; Gasperi, F.; Torri, L.; Aprea, E.; et al. Exploring influences on food choice in a large population sample: The Italian Taste project. Food Qual. Prefer. 2017, 59, 123-140. [CrossRef]

8. Meiselman, H.L.; King, S.C.; Gillette, M. The demographics of neophobia in a large commercial US sample. Food Qual. Prefer. 2010, 21, 893-897. [CrossRef]

9. Knaapila, A.; Silventoinen, K.; Broms, U.; Rose, R.J.; Perola, M.; Kaprio, J.; Tuorila, H.M. Food Neophobia in Young Adults: Genetic Architecture and Relation to Personality, Pleasantness and Use Frequency of Foods, and Body Mass Index-A Twin Study. Behav. Genet. 2011, 41, 512-521. [CrossRef]

10. Asperin, A.E.; Phillips, W.J.; Wolfe, K. Exploring Food Neophobia and Perceptions of Ethnic Foods: The Case of Chinese and Thai Cuisines. In Proceedings of the International CHRIE Conference-Refereed Track 2011 ICHRIE Conference, Denver, CO, USA, 29 July 2011.

11. Henriques, A.S.; King, S.C.; Meiselman, H.L. Consumer segmentation based on food neophobia and its application to product development. Food Qual. Prefer. 2009, 20, 83-91. [CrossRef]

12. De Toffoli, A.; Spinelli, S.; Monteleone, E.; Arena, E.; Di Monaco, R.; Endrizzi, I.; Gallina Toschi, T.; Laureati, M.; Napolitano, F.; Torri, L.; et al. Influences of Psychological Traits and PROP Taster Status on Familiarity with and Choice of Phenol-Rich Foods and Beverages. Nutrients 2019, 11, 1329. [CrossRef] [PubMed]

13. Laureati, M.; Spinelli, S.; Monteleone, E.; Dinnella, C.; Prescott, J.; Cattaneo, C.; Proserpio, C.; De Toffoli, A.; Gasperi, F.; Endrizzi, I.; et al. Associations between food neophobia and responsiveness to "warning" chemosensory sensations in food products in a large population sample. Food Qual. Prefer. 2018, 68, 113-124. [CrossRef]

14. Mustonen, S.; Oerlemans, P.; Tuorila, H. Familiarity with and affective responses to foods in 8-11-year-old children. The role of food neophobia and parental education. Appetite 2012, 58, 777-780. [CrossRef]

15. Raudenbush, B.; Frank, R.A. Assessing Food Neophobia: The Role of Stimulus Familiarity. Appetite 1999, 32, 261-271. [CrossRef] [PubMed]

16. Siegrist, M.; Hartmann, C.; Keller, C. Antecedents of food neophobia and its association with eating behavior and food choices. Food Qual. Prefer. 2013, 30, 293-298. [CrossRef]

17. Tuorila, H.; Lahteenmaki, L.; Pohjalainen, L.; Lotti, L. Food neophobia among the Finns and related responses to familiar and unfamiliar foods. Food Qual. Prefer. 2001, 12, 29-37. [CrossRef]

18. Pliner, P.; Pelchat, M.L. Neophobia in humans and the special status of foods of animal origin. Appetite 1991, 16, 205-218. [CrossRef]

19. Rozin, E.; Rozin, P. Culinary themes and variations. Nat. Hist. 1981, 90, 6-14.

20. Knaapila, A.; Tuorila, H.; Silventoinen, K.; Keskitalo, K.; Kallela, M.; Wessman, M.; Peltonen, L.; Cherkas, L.F.; Spector, T.D.; Perola, M. Food neophobia shows heritable variation in humans. Physiol. Behav. 2007, 91, 573-578. [CrossRef]

21. Duffy, E. The psychological significance of the concept of "arousal" or "activation". Psychol. Rev. 1957, 64, 265-275. [CrossRef]

22. Russell, J.A.; Weiss, A.; Mendelsohn, G.A. Affect Grid: A Single-Item Scale of Pleasure and Arousal. J. Personal. Soc. Psychol. 1989, 57, 493-502. [CrossRef]

23. Berlyne, D.E. Novelty, complexity, and hedonic value. Percept. Psychophys. 1970, 8, 279-286. [CrossRef]

24. Pliner, P.; Pelchat, M.; Grabski, M. Reduction of neophobia in humans by exposure to novel foods. Appetite 1993, 20, 111-123. [CrossRef] [PubMed]

25. Raudenbush, B.; Capiola, A. Physiological responses of food neophobics and food neophilics to food and non-food stimuli. Appetite 2012, 58, 1106-1108. [CrossRef] [PubMed]

26. Coulthard, H.; Blissett, J. Fruit and vegetable consumption in children and their mothers. Moderating effects of child sensory sensitivity. Appetite 2009, 52, 410-415. [CrossRef] [PubMed]

27. Raudenbush, B.; Schroth, F.; Reilley, S.; Frank, R.A. Food neophobia, odor evaluation and exploratory sniffing behavior. Appetite 1998, 31, 171-183. [CrossRef] [PubMed]

28. Giacalone, D.; Duerlund, M.; Boegh-Petersen, J.; Bredie, W.L.; Frost, M.B. Stimulus collative properties and consumers' flavor preferences. Appetite 2014, 77, 20-30. [CrossRef] [PubMed]

29. Spinelli, S.; De Toffoli, A.; Dinnella, C.; Monteleone, E.; Gavazzi, G.; Prescott, J.; Pierguidi, L. Individual differences in arousal induced by taste quality, intensity and valence. In Proceedings of the 9th European Conference on Sensory and Consumer Science, Rotterdam, The Netherlands, 13-15 December 2020.

30. Olabi, A.; Neuhaus, T.; Bustos, R.; Cook-Camacho, M.; Corvi, T.; Abdouni, L. An investigation of flavor complexity and food neophobia. Food Qual. Prefer. 2015, 42, 123-129. [CrossRef]

31. Pliner, P.; Melo, N. Food Neophobia in Humans: Effects of Manipulated Arousal and Individual Differences in Sensation Seeking. Physiol. Behav. 1997, 61, 331-335. [CrossRef] 
32. Zuckerman, M.; Kolin, E.A.; Price, L.; Zoob, I. Development of a Sensation-Seeking Scale. J. Consult. Psychol. 1964, $28,477-482$. [CrossRef]

33. Tuorila, H.; Meiselman, H.L.; Bell, R.; Cardello, A.V.; Johnson, W. Role of sensory and cognitive information in the enhancement of certainty and liking for novel and familiar foods. Appetite 1994, 23, 231-246. [CrossRef]

34. Moding, K.J.; Stifter, C.A. Temperamental approach/withdrawal and food neophobia in early childhood: Concurrent and longitudinal associations. Appetite 2016, 107, 654-662. [CrossRef] [PubMed]

35. Alley, T.R.; Potter, K.A. Food Neophobia and Sensation Seeking. In Handbook of Behavior, Food and Nutrition; Preedy, V., Watson, R., Martin, C., Eds.; Springer: Berlin/Heidelberg, Germany, 2011; pp. 707-724.

36. Brown, S.D.; Harris, G. A Theoretical Proposal for a Perceptually Driven, Food-Based Disgust that Can Influence Food Acceptance During Early Childhood. Int. J. Child Health Nutr. 2012, 1, 1-10. [CrossRef]

37. Spinelli, S.; De Toffoli, A.; Dinnella, C.; Laureati, M.; Pagliarini, E.; Bendini, A.; Braghieri, A.; Gallina Toschi, T.; Sinesio, F.; Torri, L.; et al. Personality traits and gender influence liking and choice of food pungency. Food Qual. Prefer. 2018, 66, 113-126. [CrossRef]

38. Mascarello, G.; Pinto, A.; Rizzoli, V.; Tiozzo, B.; Crovato, S.; Ravarotto, L. Ethnic Food Consumption in Italy: The Role of Food Neophobia and Openness to Different Cultures. Foods 2020, 9, 112. [CrossRef]

39. Farrow, C.V.; Coulthard, H. Relationships between sensory sensitivity, anxiety and selective eating in children. Appetite 2012, 58, 842-846. [CrossRef]

40. Zickgraf, H.F.; Elkins, A. Sensory sensitivity mediates the relationship between anxiety and picky eating in children/ adolescents ages 8-17, and in college undergraduates: A replication and age-upward extension. Appetite 2018, 128, 333-339. [CrossRef]

41. Maratos, F.A.; Staples, P. Attentional biases towards familiar and unfamiliar foods in children. The role of food neophobia. Appetite 2015, 91, 220-225. [CrossRef] [PubMed]

42. Cisler, J.M.; Koster, E.H. Mechanisms of attentional biases towards threat in anxiety disorders: An integrative review. Clin. Psychol. Rev. 2010, 30, 203-216. [CrossRef]

43. Jaeger, S.R.; Chheang, S.L.; Jin, D.; Ryan, G.; Worch, T. The negative influence of food neophobia on food and beverage liking: Time to look beyond extreme groups analysis? Food Qual. Prefer. 2021, 92, 104217. [CrossRef]

44. Ritchey, P.N.; Frank, R.A.; Hursti, U.-K.; Tuorila, H. Validation and cross-national comparison of the food neophobia scale (FNS) using confirmatory factor analysis. Appetite 2003, 40, 163-173. [CrossRef]

45. Peryam, D.R.; Pilgrim, F.J. Hedonic scale method of measuring food preferences. Food Technol. 1957, 11, 9-14.

46. Jaeger, S.R.; Roigard, C.M.; Hunter, D.C.; Worch, T. Importance of food choice motives vary with degree of food neophobia. Appetite 2020, 159, 105056. [CrossRef] [PubMed]

47. Jaeger, S.R.; Roigard, C.M.; Le Blond, M.; Hedderley, D.I.; Giacalone, D. Perceived situational appropriateness for foods and beverages: Consumer segmentation and relationship with stated liking. Food Qual. Prefer. 2019, 78, 103701. [CrossRef]

48. Jaeger, S.R.; Prescott, J.; Worch, T. Food neophobia modulates importance of food choice motives: Replication, extension and behavioural validation. Food Qual. Prefer. 2021, 96, 104439.

49. Tavakol, M.; Dennick, R. Making sense of Cronbach's alpha. Int. J. Med. Educ. 2011, 2, 53-55. [CrossRef]

50. Schickenberg, B.; van Assema, P.; Brug, J.; de Vries, N.K. Are the Dutch acquainted with and willing to try healthful food products? The role of food neophobia. Public Health Nutr. 2007, 11, 493-500. [CrossRef]

51. Verbeke, W. Consumer acceptance of functional foods: Socio-demographic, cognitive and attitudinal determinants. Food Qual. Prefer. 2005, 16, 45-57. [CrossRef]

52. Jacobs, S.; Sioen, I.; Pieniak, Z.; De Henauw, S.; Maulvault, A.L.; Reuver, M.; Fait, G.; Cano-Sancho, G.; Verbeke, W. Consumers' health risk-benefit perception of seafood and attitude toward the marine environment: Insights from five European countries. Environ. Res. 2015, 143, 11-19. [CrossRef]

53. Burger, J.; Gochfeld, M. Perceptions of the risks and benefits of fish consumption: Individual choices to reduce risk and increase health benefits. Environ. Res. 2009, 109, 343-349. [CrossRef]

54. Pieniak, Z.; Verbeke, W.; Scholderer, J.; Brunsø, K.; Ottar Olsen, S. Impact of consumers' health beliefs, health involvement and risk perception on fish consumption. Br. Food J. 2008, 110, 898-915. [CrossRef]

55. Rozin, P. Social learning about food by Humans. In Social Learning: Psychological and Biological Perspectives; Zentall, T.R., Galef, B.G., Eds.; Erlbaum: Hillsdale, NJ, USA, 1988; pp. 165-187.

56. Sulmont-Rossé, C.; Chabanet, C.; Issanchou, S.; Köster, E.P. Impact of the arousal potential of uncommon drinks on the repeated exposure effect. Food Qual. Prefer. 2008, 19, 412-420. [CrossRef]

57. Kraig, B.; Sen, C.T. Street Food around the World: An Encyclopedia of Food and Culture; ABC-CLIO: Santa Barbara, CA, USA, 2013.

58. Ireland, J.; van Erp-Baart, A.; Charrondière, U.; Møller, A.; Smithers, G.; Trichopoulou, A. Selection of a food classification system and a food composition database for future food consumption surveys. Eur. J. Clin. Nutr. 2002, 56, S33-S45. [CrossRef]

59. Feldman Barrett, L.; Russell, J.A. Independence and bipolarity in the structure of current affect. J. Personal. Soc. Psychol. 1998, 74, 967-984. [CrossRef]

60. den Uijl, L.C.; Jager, G.; Zandstra, E.H.; de Graaf, C.; Kremer, S. Self-reported food-evoked emotions of younger adults, older normosmic adults, and older hyposmic adults as measured using the PrEmo2 tool and the Affect Grid. Food Qual. Prefer. 2016, 51, 109-117. [CrossRef] 